\title{
Tiempos de cambio para las enfermeras de salud mental en España
}

Times of Change for Mental Health Nurses in Spain

\section{Montserrat García Sastre}

Universidad de Alcalá, Departamento de Enfermería. Alcalá de Henares, España.

Contacto: mmontserrat.garcia@uah.es

Fecha de recepción: 11 de noviembre de 2018 / Fecha de aceptación: 19 de diciembre de 2018

Hace unos meses, concretamente el 18 de mayo de 2019, se reunieron en el salón de actos Ernest Lluch del Ministerio de Sanidad, Servicios Sociales e Igualdad en Madrid, un número significativo de enfermeras de salud mental con motivo de celebrar la $2^{\text {a }}$ Conferencia Estatal de Enfermería de Salud Mental y la $6^{\text {a }}$ Sesión del Seminario Permanente de Jóvenes Investigadores en Cuidados de Salud Mental organizada por la Asociación Española de Enfermería de Salud Mental (AEESME).

El acto, de reconocido interés científico y profesional, fue especialmente entrañable y productivo para las enfermeras de salud mental reunidas: referentes de considerable prestigio social, jóvenes promesas y residentes en formación. Como no podía ser de otro modo, el acto transcurrió en un clima de diálogo y consenso disciplinar que propició una considerable producción de ideas y reflexiones con miras de cambio y mejora.

Tanto el grupo de Pensadores como el vivero de Jóvenes Investigadores debatieron sobre la situación actual de la Enfermería de Salud Mental en materia de formación, investigación y desarrollo profesional durante una larga jornada de trabajo.

Se inició el debate a partir de una exposición de los antecedentes históricos y del marco conceptual de la Especialidad de Enfermería de Salud Mental, reseñándose los hitos más relevantes, desde la regulación de la especialidad en la década de los años 70, el cambio del 98 en el marco de la formación especializada mediante el sistema de residencia de un año de duración y el actual sistema de formación de dos años aprobado en el año 2011.

La Especialidad no solo ha evolucionado a partir de estos hitos, sino que viene siendo influenciada, en lo positivo y en lo negativo, por la evolución de la propia disciplina y de las propias enfermeras, el sistema sociosanitario, los cambios sociales, demográficos y epidemiológicos, la crisis económica, las actuales políticas de salud y los avances científico-tecnológicos...

De entre todo ello, destacar el empoderamiento demostrado y la proactividad de las personas con sufrimiento psíquico, demostrando una participación y un dinamismo imprescindible en pro de la recuperación y los derechos humanos.

Vienen aconteciendo avances y retrocesos, depositando una sensación agridulce generalizada en las enfermeras especialistas de salud mental. Son tiempos de esperanza e ilusión, pero simultáneamente también de mucha frustración e incertidumbre.

Las enfermeras de salud mental de la AEESME no nos conformamos y, por tanto, queremos gestionar un futuro diferente. No se avecinan, lo son ya, tiempos de cambio para las enfermeras de salud mental en España. Cambios por y para promover la salud mental de la población desde un cuidado integral, cambios por y para las personas y familias que necesitan cuidados para recuperar su salud y restablecer la salud y el funcionamiento familiar. Cambios orientados a la comunidad, pues solo desde ahí se construye la verdadera salud pública. Cambios para ellas, con ellas. 
Inevitablemente, los cambios transitan a partir de la formación, en este caso de las enfermeras de salud mental y la transformación del sistema. En el contexto de la formación y el desarrollo actual de la especialización, se han sucedido cambios profundos. Un cambio determinante ha sido el cambio de denominación, pasando a llamarse Enfermería de Salud Mental versus Enfermería Psiquiátrica... Este cambio nominativo implica no solo traspasar el modelo tradicional biomédico, sino ampliar la orientación de los cuidados hacia la salud positiva, concretamente la promoción de la salud y la prevención de problemas.

Otro cambio importante fue la modificación del programa formativo, la redefinición de competencias y, por ende, la ampliación de la duración de la formación a dos años, incrementándose las oportunidades de formación y la integración de aprendizajes de manera más reflexiva, sosegada.

En estos últimos años parecería que nos encontramos en una situación más favorable, pero la pregunta es... ¿Se está consiguiendo de la manera prevista y extensivamente por igual en todo el territorio español? Las voces enfermeras, allí en el salón de actos del Ministerio, prácticamente al unísono coincidieron en que no...

Existen debilidades y amenazas importantes. ¿Somos las enfermeras lo suficientemente conscientes de las necesidades de salud de las personas y atendemos las demandas de cuidado? ¿Estamos lo suficientemente cualificadas tanto desde el marco conceptual como técnico de los cuidados? ¿Existe verdaderamente compromiso por parte de la profesión para la mejora continua? ¿El sistema permite que las enfermeras desarrollen todo su potencial? ¿Las propias enfermeras estamos realmente comprometidas?

Ante preguntas transcendentes, se necesitan respuestas desde el rigor, desde la disciplina y la especialidad fundamentadas en procesos de razonamiento crítico serios y responsables... Y de esto, esta jornada, gracias a la colaboración de todo el aforo, se impregnó.

Las enfermeras de salud mental avanzamos, pero despacio, parecería incluso que con cierto retraso... Vamos al ritmo que a la Especialidad se le permite avanzar.

En el marco de nuestra asociación, la AEESME, seguimos pensando, imaginando y construyendo un futuro que garantice mayor calidad... Mayor calidad de vida para las personas/familias, la sociedad en general y para las enfermeras de salud mental en particular, mayor calidad asistencial y docente.

Es necesario gestionar el cambio, y este pasa por potenciar la investigación, la innovación y la gestión a partir de un paradigma sólido que permita cuidar desde la esencia del cuidado respetuoso, desde las $5 \mathrm{C}$ del cuidado descritas por Roach: Compasión, Competencia, Compromiso, Comunicación y Confianza. Las enfermeras hemos de ser sensibles, hemos de demostrar competencia, constancia, cuidar la relación terapéutica... hemos de ser confiables y hemos de confiar en nosotras mismas.

Pensadores y Jóvenes Investigadores coinciden en las siguientes premisas para el cambio que han de marcar la agenda de nuestra Especialidad:

- Las enfermeras hemos de establecer un compromiso de cuidados integrales y eficientes para fortalecer la capacidad de autocuidado de las personas, desde la perspectiva de la recuperación y en el medio comunitario. Reorientar el sistema de atención sociosanitaria, traspasar el modelo biomédico tradicional a un modelo salutogénico fundamentado en la Promoción de la Salud.

- Las enfermeras asumiremos el liderazgo que nos corresponde en todos los ámbitos de nuestra profesión, incluida la gestión, traspasando las limitaciones del sistema, demostrando nuestra competencia y cualificación en beneficio de la salud de las personas. Lo que A. Rigol manifestó como: "Las enfermeras tenemos mucho poder, se hace camino andando, hemos de ser capaces de cambiar las instituciones, hay que empoderar a las personas, empoderarnos a nosotros mismos". 
- La formación en la especialidad ha de cumplir unos mínimos estándares y criterios de calidad adaptándose al devenir de los tiempos y las necesidades de las personas. La evaluación y auditoría del proceso formativo y la estructura organizativa, véase la unidad docente, ha de realizarse en pro de la mejora de la calidad docente y el desarrollo de la Especialidad. Existe acuerdo en afirmar que la implementación del programa formativo en las diferentes unidades docentes es desigual, incluso vulnerando en ocasiones los derechos formativos de los residentes, se orienta más hacia la enfermedad que hacia la promoción y no siempre garantiza la participación activa de los residentes en su propio proceso. Además, se observan diferencias notables respecto a la docencia y formación continuada (incluidas sesiones clínicas y oportunidades de asistencia a actos científicos), cultura de investigación, protocolo de supervisión y evaluación con criterios diferentes respecto a la realización de proyectos o estudios de investigación, individuales o en grupo.

- Parece también conveniente clarificar de manera efectiva el rol del residente, la enfermera en formación especializada debe tener garantizada una supervisión y tutoría continuada, priorizándose la adquisición de competencias en materia de investigación. El residente ha de recibir una formación específica enfermera e interdisciplinar, asumiendo su rol activo en este proceso formativo, así como tiene que ver garantizados derechos laborales.

- El reconocimiento de la coordinación docente y la acción tutorial de las enfermeras que posibilitan la formación en las más de ochenta unidades docentes de todo el territorio español también es una necesidad para garantizar la calidad del proceso. La formación continuada de estos profesionales es imprescindible, debiéndose incidir igualmente en la competencia investigadora.

- El actual programa de formación de la Especialidad aprobado en el año 2011 debiera revisarse con carácter periódico. Parece que es momento de comenzar e incluso reflexionar sobre si procede la ampliación del periodo de formación. Evidentemente, para concluir, se considera que es imprescindible, y por imprescindible inaplazable, el reconocimiento de la creación de la categoría profesional y la catalogación de puestos de trabajo en todo el territorio español. La atención en todos los dispositivos especializados en salud mental, independientemente de su titularidad (pública o privada) debe ser prestada por especialistas.

De todo ello, con calma y rigor, las enfermeras pensaron y dialogaron en esta $2^{\mathrm{a}}$ Conferencia Estatal de Enfermería de Salud Mental para cambiar el presente hacia un futuro de salud mental en positivo. 